\title{
Comparative analysis of portable image capture devices for photo-documentation in otology clinics
}

\author{
Daniel Moualed, Olivia Whiteside, Chris Aldren
}

John Radcliffe Hospital, Oxford \& Wexham Park Hospital, Slough

Background: The use of photo-documentation in ENT clinics is becoming more common as technological advances have made the hardware required more accessible. Otoscopy photographs provide a more accurate record of tympanic membranes than hand-drawn diagrams and should be regarded as the gold-standard in documentation.

Aim: To systematically review and compare a range of otoscopy image capture devices in a simulated clinical environment.

Methods: Five commercially available products were acquired for review: Endoscope-i, Cupris TYM, Firefly DE1250, Syncvision, and Nikon P5100. Three ENT surgeons tested the devices on each other and independently rated the products for ease of use, and field of view. The best images captured were then collected (6 ears for each device), randomised, and distributed to five ENT surgeons for independent and 'blinded' grading of image quality.

Results: The best images were obtained with the Endoscope-i (+ iPhone 7) and Syncvision systems, while the Nikon and Firefly also provided very good quality images. The Cupris TYM is the only product on test not to utilise an endoscope, and the image quality suffers as a result. The most ergonomic and easy to use products were the Syncvision and Nikon camera with endoscope adapters.

Cupris - TYM

Type: Smartphone otoscope adapter for iPhone $5 / 6 \quad$ Cost: $£ 129$

Image quality: $32 \%$ Field of view: $42 \%$ Ease of use: $58 \%$

Comments: The images taken were mostly too dark and lacking in colour (tested with iPhone 6s). The picture was too zoomed in due to a poor field of view which made it difficult to navigate the tympanic membrane and get representative images. This product does have the advantage of not requiring any additional equipment such as endoscopes or light sources which is useful in low resource settings.

\section{Endoscope-i **BEST VALUE**}

Type: Endoscope adapter for iPhone 5/6/7/8/X Cost: $£ 99$ Image quality: $87 \%$ Field of view: $100 \%$ Ease of use: $58 \%$

Comments: Overall excellent image quality and field of view (tested with iPhone 7). Somewhat awkward for photo use due to the distance of the capture button from the endoscope attachment position, this resulted in a tendency for movement at the moment of photo taking. This was not a problem for videos which could be started and then a more comfortable grip found.

\section{Firefly - DE1250}

Type: Wireless endoscopic camera Cost: $£ 780$ Image quality: $75 \%$ Field of view: $100 \%$ Ease of use: $71 \%$

Comments: Good image quality, but also does not have the image capture button in the ideal position as it is too far from endoscope attachment. The live stream to laptop feature is a useful aid to communication as it allows images or videos to be projected in real time on to a screen visible to the patient during the examination.

\section{Digital camera + adapters}

Type: Nikon P5100 Compact (12 megapixel) Cost: Ur-e20 adapter $=£ 49$, Storz 590-70 coupling $=£ 172$ Image quality: $80 \%$ Field of view: $100 \%$ Ease of use: $95 \%$

Comments: This camera model is no longer available but was included in the test for comparative purposes as it was already in use in our department. Adapters can be purchased for most digital cameras and offer an alternative to bespoke products. Digital cameras provide very good image quality and are exceptionally easy to use due to ergonomic and intuitive camera controls.

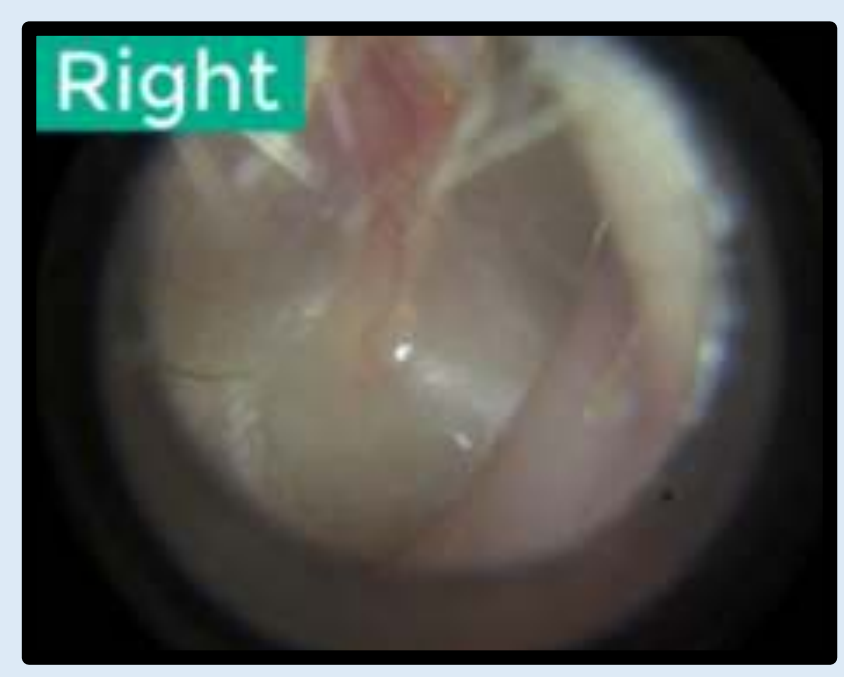

\section{Syncvision - iO1 OTO **BEST TESTED**}

Type: Complete system Cost: $£ 780$ (incl. shipping + tax)

Image quality: $85 \%$ Field of view: $93 \%$ Ease of use: $93 \%$

Comments: Excellent image quality, and very easy to use. The Syncvision is designed to be held vertically, although holding it horizontally as for an otoscope feels more natural. Very quick to set up and use, capture button conveniently placed. Has the advantage of not requiring any additional equipment. 